\title{
Awake craniotomy for glioblastoma in COVID-19-positive patients and delivering the standard of care: illustrative case
}

\author{
Reinier Alvarez, MD, ${ }^{1,2}$ Rupesh Kotecha, MD, ${ }^{1,3}$ Michael W. McDermott, MD, ${ }^{1,4}$ and Vitaly Siomin, $\mathrm{MD}^{3,4}$ \\ ${ }^{1}$ Herbert Wertheim College of Medicine, Florida International University, Miami, Florida; ${ }^{2}$ Department of Neurosurgery, University of Colorado School of Medicine, Aurora, \\ Colorado; ${ }^{3}$ Department of Radiation Oncology, Miami Cancer Institute, Baptist Health South Florida, Miami, Florida; and ${ }^{4}$ Department of Neurosurgery, Miami Neuroscience \\ Institute, Baptist Health South Florida, Miami, Florida
}

\begin{abstract}
BACKGROUND Providing the standard of care to patients with glioblastoma (GBM) during the novel coronavirus of 2019 (COVID-19) pandemic is a challenge, particularly if a patient tests positive for severe acute respiratory syndrome coronavirus 2 (SARS-CoV-2). Further difficulties occur in eloquent cortex tumors because awake speech mapping can theoretically aerosolize viral particles and expose staff. Moreover, microscopic neurosurgery has become difficult because the use of airborne-level personal protective equipment (PPE) crowds the space between the surgeon and the eyepiece. However, delivering substandard care will inevitably lead to disease progression and poor outcomes.
\end{abstract}

OBSERVATIONS A 60-year-old man with a left insular and frontal operculum GBM was found to be COVID-19 positive. Treatment was postponed pending a negative SARS-CoV-2 result, but in the interim, he developed intratumoral hemorrhage with progressive expressive aphasia. Because the tumor was causing dominant hemisphere language symptomatology, an awake craniotomy was the recommended surgical approach. With the use of airborne-level PPE and a surgical drape to protect the surgeon from the direction of potential aerosolization, near-total gross resection was achieved.

LESSONS Delaying the treatment of patients with GBM who test positive for COVID-19 will lead to further neurological deterioration. Optimal and timely treatment such as awake speech mapping for COVID-19-positive patients with GBM can be provided safely.

https://thejns.org/doi/abs/10.3171/CASE21246

KEYWORDS glioblastoma; awake craniotomy; COVID-19; neuro-oncology

The novel coronavirus pandemic of 2019 (COVID-19) caused by severe acute respiratory syndrome coronavirus 2 (SARS-CoV-2) has disrupted various aspects of life and introduced obstacles in the delivery of non-COVID-related healthcare. ${ }^{1,2}$ Providing the appropriate care to neuro-oncology patients while minimizing potential COVID-19 exposure remains an evolving topic of discussion. A delay in treatment will inevitably lead to progression of disease, particularly in patients with high-grade malignancies, such as glioblastoma (GBM). Awake craniotomy with functional mapping to ensure safe resection has become a standard initial treatment for tumors located in the speech language cortex. ${ }^{3}$ However, the concern for potential SARS-CoV-2 aerosolization and subsequent risk of viral transmission during an awake craniotomy on a patient with COVID-19 may lead to a delay in providing adequate care and present an obstacle for the treatment team. The use of personal protective equipment (PPE) in the time of COVID-19 also creates a further impediment because it crowds the space between the surgeon and the binoculars of the microscope. ${ }^{4,5}$

Ensuring the safety of involved healthcare workers is undoubtedly a priority but should not come at the expense of providing adequate patient care. As such, various recommendations for providing safe surgical treatment for patients with COVID-19 have been published since the onset of the pandemic, although recommendations on clinical decision-making for awake language mapping surgery on patients with COVID-19- have not reached widespread consensus. ${ }^{6-16}$

ABBREVIATIONS CDC = Centers for Disease Control and Prevention; $C T=$ computed tomography; FLAIR = fluid-attenuated inversion recovery; FOV = field of view; $\mathrm{GBM}=$ glioblastoma; IMRT = intensity-modulated radiation therapy; MRI = magnetic resonance imaging; PAPR = personal air purification respirators; PPE = personal protective equipment; SARS-CoV-2 = severe acute respiratory syndrome coronavirus 2; TMZ = temozolomide.

INCLUDE WHEN CITING Published July 5, 2021; DOI: 10.3171/CASE21246.

RECEIVED April 19, 2021. ACCEPTED May 5, 2021.

(C) 2021 The authors, CC BY-NC-ND 4.0 (http://creativecommons.org/licenses/by-nc-nd/4.0/). 
To our knowledge, and based on a review of the published literature (until April 2021), only one case of an awake craniotomy for a GBM on a patient with COVID-19 has been reported in the literature. ${ }^{17}$ Here we present an additional case of a patient with GBM, who tested positive for COVID-19, involving the eloquent cortex complicated by intratumoral hemorrhage and midline shift that required an awake resection. Because challenges to providing care for neurosurgical patients will persist during the COVID-19 pandemic and its aftermath, the objective of this report is to suggest that high-quality standards should not be compromised because this group of patients can be treated safely and expeditiously.

\section{Illustrative Case}

\section{Clinical Presentation}

A 60-year-old, right-handed man presented to his primary care physician with a 4-month history of right-sided tinnitus and hearing intermittent loud noises that he described as "bells and whistles." Magnetic resonance imaging (MRI) revealed a patchy infiltrative signal abnormality within the left insular region and portions of the left frontal operculum, suggestive of neoplastic disease. He was referred to the neurosurgery service and was then found to be COVID-19 positive 10 days later. At that time, South Florida was a COVID-19 hotspot. Following the recommendations of our institution to control COVID-19 infection, his diagnostic testing was put on hold because he was not considered to require emergency treatment.

Approximately 1 month after initial diagnosis, he presented to an outside hospital with complaints of worsening headache, difficulty with balance, new-onset expressive aphasia, and confusion. SARSCoV-2 reverse transcription polymerase chain reaction (RT-PCR) remained positive, and a noncontrast head computed tomography (CT) scans showed a large mass in the left temporal lobe with an acute $1.6 \times 0.9 \times 1.5-\mathrm{cm}$ intratumoral hemorrhage, surrounding edema, mass effect on the left lateral ventricle, and minimal midline shift. He was transferred to our institution for evaluation and was found to have a positive pronator drift on the right side as well as diminished grip and intrinsic strength compared to the left side. Brain MRI showed a left temporoparietal lobe-enhancing mass measuring $3.5 \times 3.1 \times 3.2 \mathrm{~cm}$ with necrotic and hemorrhagic components that surrounded an infiltrative and nonenhancing component. Perfusion imaging showed increased cerebral blood volume within the enhancing portion of the tumor consistent with a malignant neoplasm. MR spectroscopy demonstrated elevated choline peak to creatinine ratio $>2: 1$ in the enhancing portion as well as depressed $\mathrm{N}$-acetylaspartate. MRI fluid-attenuated inversion recovery (FLAIR) showed additional infiltrative signal without enhancement extending into the temporal lobe, left basal ganglionic capsule, thalamus, and midbrain and extending superiorly into the parietal and posterior frontal lobes.

During his hospital course, the patient experienced progression of his aphasia with further word-finding difficulty. His worsening symptoms and evidence of mass effect were concerning and continued to progress. Because of concern regarding possible rapid neurological decline, we recommended resection despite his COVID-19-positive status. Considering the left temporal-occipital location and dominant hemisphere language symptomatology, we believed that an awake craniotomy was a prudent surgical approach. However, the patient's positive COVID-19 status added an extra layer of complexity to the logistics of the surgery.

\section{Treatment Course}

Our institution's COVID-19 PPE requirements consisted of wearing an N95 respirator mask covered with a surgical mask and eye protection (goggles or a face shield). Individuals involved in surgeries of patients with COVID-19 were required to wear personal air purification respirators (PAPR), such as the Flyte system (Stryker). However, because the Flyte helmet served as an impediment to the proper use of the operating microscope, the operating surgeon believed that it could lead to a subpar resection and possibly worse outcome. The administration allowed the surgeon to follow the standard airbornelevel precaution ${ }^{18}$ with PPE consisting of an N95 mask covered by a surgical mask to facilitate use of the microscope while all other members of the operating room staff used the Stryker Flyte system (Fig. $1 \mathrm{~A}$ and $\mathrm{B})$. The patient wore a procedure mask when not involved in any neurocognitive testing. The surgeon was separated from the vicinity of the patient's face by a large, clear plastic drape to prevent any potential viral aerosols from traveling in the surgeon's direction (Fig. 1C). A sleep-awake-sleep protocol was implemented with a laryngeal mask airway. The awake craniotomy proceeded with no intraoperative complications and near-total resection, and pathology was consistent with a high-grade glioma (isocitrate dehydrogenase wild-type, $O$ (6)-methylguanine DNA methyltransferase unmethylated, negative for ATP-dependent helicase, or epidermal growth factor receptor VIII mutations). The patient had an uneventful postoperative course, and notable improvement in speech was evident with
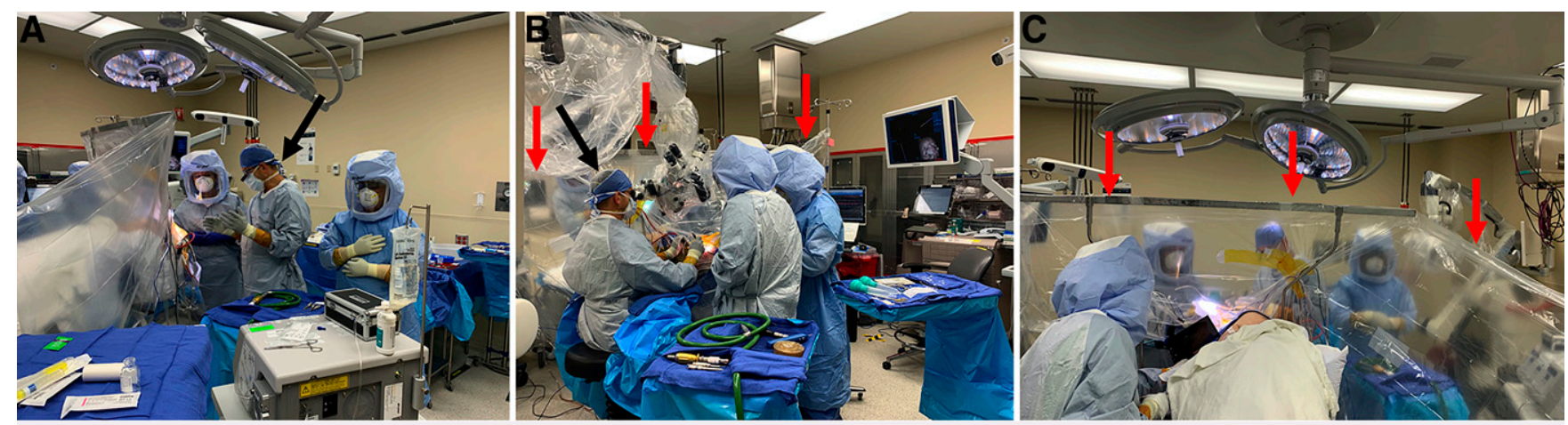

FIG. 1. Operating room logistics. A: Surgeon (black arrow) with N95 respirator covered with a surgical mask in sterile area while other operating room personnel use a PAPR. B: Surgeon using operating microscope (black arrow) in sterile area separated from patient with clear drape (red arrows). C: Clear surgical drape (red arrows) separating the sterile area and preventing viral aerosolization from traveling toward the surgeon. 



FIG. 2. Gadolinium-enhanced T1 MRI of a patient with GBM. Preoperative gadolinium-enhanced axial MRI (A), immediate postoperative axial MRI (B), and 5-month postoperative axial MRI (C). D-F: Sagittal MRIs corresponding to time points of the axial images.

diminished expressive aphasia. He was discharged home on postoperative day 4 with a corticosteroid taper.

SARS-CoV-2-negative RT-PCR was documented 3 weeks postoperatively; however, at that time, repeat imaging was consistent with tumor recurrence. A new centrally necrotic mass with peripheral enhancement was found to encase the prior surgical bed, causing significant edema and worsening midline shift. The patient received $60 \mathrm{~Gy}$ of intensity-modulated radiation therapy (IMRT) in 30 fractions with temozolomide (TMZ) and began adjuvant TTFields therapy (Optune). He remained free of neurological symptoms for 5 weeks after completion of IMRT but then presented to the emergency department with complaints of worsening right-sided weakness and new-onset left frontal headaches. Repeat brain MRI showed progression of the left frontotemporal mass with increased vasogenic edema and increased cerebral blood volume consistent with a mixture of radiation necrosis and residual tumor after prior near-total resection (Fig. 2). The patient was subsequently restarted on TMZ with concurrent TTFields therapy. After two cycles of TMZ, repeat imaging at 5 months postoperatively showed stable enhancement, necrosis, and perfusion but with slight increase in FLAIR signal, again favoring mixed necrosis with residual tumor. Repeat surgery was not recommended at that time, and he was placed on palliative bevacizumab therapy.

\section{Discussion}

GBM is the most common primary malignant brain tumor, with an incidence rate of 3.19 per $100,000 .^{19}$ The COVID-19 pandemic has added an extra layer of complexity to providing timely neurosurgical care for patients with GBM. Treatment for high-grade gliomas is not usually performed on an emergency basis, but prompt surgical treatment within 2 weeks is recommended. ${ }^{20}$ During the pandemic, this time frame may be difficult to follow because patients may present with COVID-19-positive status, leading treatment teams to wait for a negative SARS-CoV-2 RT-PCR. However, tumor-related complications such as intracranial hemorrhage ${ }^{21}$ or status epilepticus ${ }^{22}$ may present with accelerated neurological decline that requires urgent surgical decompression despite a patient's COVID-19 status. Here we present the case of a patient with COVID-19 whose treatment was initially postponed but then required an urgent awake craniotomy for tumor progression. This case is a testament to the fact that performing an awake speech mapping craniotomy for these patients is possible.

The Centers for Disease Control and Prevention (CDC) has been at the forefront of establishing recommendations on how to provide healthcare during COVID-19. However, the CDC has delegated most restrictions on healthcare to local health departments and individual institutions. ${ }^{23}$ The recommendations are based on 
the potential for patient harm if treatment is delayed as well as the risk for healthcare workers and community transmission. As with all healthcare decisions, the clinical judgment of medical providers is of utmost importance in determining the appropriate subsequent steps and associated health risks. Because COVID-19 has infiltrated all aspects of healthcare and will continue to do so for an unknown amount of time, it is important that we continue to provide the standard quality of care to all patients, including those with neuro-oncological disease.

The goals of neurosurgical intervention are to reduce mass effect, acquire tissue for molecular and histological identification, and achieve cytoreduction. ${ }^{24}$ After resection, patients are treated with adjuvant chemotherapy and radiotherapy. ${ }^{24,25}$ Special precautions are necessary during outpatient radiotherapy, including changes to patient selection for radiotherapy treatment, ${ }^{26}$ consideration of hypofraction for elderly patients, ${ }^{26}$ screening for COVID-19 before CT simulation and treatment initiation, ${ }^{27}$ enhanced precautions and PPE use for therapists (because these patients cannot wear a mask and have a thermoplastic mask for immobilization), ${ }^{28}$ use of videoconference platforms to enable peer review for treatment planning, ${ }^{29}$ virtual treatment visits for weekly patient assessments, ${ }^{30}$ and disinfection of radiotherapy vaults after use. ${ }^{31}$ The surgical treatment for gliomas must include the maximum extent of resection because it is associated with improved survival ${ }^{24,32}$ and preserves neurological function. The preferred technique of resection for tumors located in eloquent areas of the brain is an awake surgical approach with cortical and subcortical mapping to ensure maximal safe resection. ${ }^{3}$ A sleepawake-sleep anesthesia protocol is commonly used to allow for patient interaction and verbalization throughout the surgery. Some centers prefer laryngeal mask anesthesia during the early part of a language mapping craniotomy; however, this technique may increase the risk of exposure for anesthesia and other operating room personnel. Using an awake approach during the COVID-19 pandemic brings about concerns of virus particle aerosolization and potential exposure to the operating room staff, which on average, may include 7 to 10 individuals. This concern is heightened especially when performing surgery on a patient with COVID19 , leading to the appropriate recommendation to delay surgery or use other diagnostic methods. ${ }^{10,15,16,33}$

Additional difficulties in providing neurosurgical care arise from the encumbrance of PPE during use of the operating microscope. The use of bulky safety goggles and/or voluminous N95 respirators crowds the space, increasing the distance between the surgeon and the eyepieces by $>5 \mathrm{~mm}^{4}$ Apart from the ergonomic disadvantage, the increased distance poses limitations on the surgeon's field of view (FOV). Limited microscopic FOV has been documented, with laboratory blast goggles and face shields noted to cause significantly diminished FOV when compared to the naked eye. ${ }^{34}$ Clamp and Broomfield showed that the use of an N95 valved respirator mask reduced the visible target area to $64 \%$; adding safety goggles further reduced the visibility to $36 \%$. ${ }^{4}$ Diminished visibility can introduce surgical error and/or suboptimal resection because reduced FOV is associated with increased errors in eye-hand coordination tasks and requires more time to complete tasks. To mitigate the potential for any surgical errors or substandard care, our operating neurosurgeon performed the surgery using an N95 respirator covered with a surgical mask and his own prescription eyeglasses. Because the patient was on the other side of a transparent drape facing the opposite direction of the surgeon, aerosolization of viral particles directed toward the surgeon was believed to be unlikely. However, there is no documented evidence of risk or lack thereof in such a situation, which may subsequently bring about a medicolegal concern regarding a surgeon's safety. The operating neurosurgeon never developed symptoms of COVID-19 infection or tested positive in subsequent months.

Modifications to operating room logistics and equipment have been reported in the literature as methods that may mitigate SARS-CoV-2 transmission and ameliorate obstacles related to microscope use. Using additional "tent" microscope drapes and patient isolation enclosures during aerosol-generating procedures provides physical barriers that may protect operating room personnel. ${ }^{35-43}$ Nonetheless, a physical barrier method can only be implemented in surgeries during which patients are fully anesthetized and intubated; thus, it is not applicable to awake neurosurgery. Recent emergence of increased applications of the three-dimensional exoscope has also provided the ability to perform microsurgery while donning full PAPR respirators, as has been suggested by the otorhinolaryngology field. ${ }^{5,44}$ However, as a relatively new piece of equipment, it is not easily accessible to all neurosurgeons and may not serve as a solution to obstacles encountered with PPE and microscope use.

\section{Observations}

In addition to the hurdles associated with providing general care to patients with COVID-19, neurosurgical care is further convoluted for neuro-oncology. Surgical care for patients with COVID-19 is now a rather common occurrence with various safety measures in place, although consensus-based recommendations on performing awake neurosurgery or proper use of the operating microscope while ensuring safety are lacking. Because the safety of all healthcare personnel is of utmost importance, prudent restrictions are in place; however, they should not compromise the care we provide to our patients. Delaying treatment of patients with GBM who test positive for SARSCoV-2 may lead to the development of tumor-related complications and neurological deficits, as in the case presented here. Because COVID-19 will persist for an unknown amount of time and continue to affect the delivery of neurosurgical care, we must adapt and ensure that we provide the standard of care.

\section{Lessons}

The field of neurosurgery needs evidence-based recommendations and further adaptations to provide the usual standard of care. Providing optimal and timely treatment for GBM, such as awake speech mapping, even in patients with COVID-19 can be performed safely and expeditiously. Our recommendations for safe awake

\section{TABLE 1. Recommendations for awake speech language} mapping craniotomy in patients with COVID-19

1. Airborne-level PPE for all operating room personnel*

2. PAPR for anesthesia, scrub nurse, neurophysiologists/ neuropsychologists, and surgical assistants

3. Clear protective drape separating the surgeon from a patient's face during neurocognitive testing

4. Avoidance of laryngeal mask or nasal airways during early parts of case

5. Postanesthetic recovery room isolation

* Airborne-level PPE includes eye protection, gown, gloves, and N95 mask or PAPR. 
speech language mapping craniotomy in patients with COVID-19 are summarized in Table 1.

\section{References}

1. Lara-Reyna J, Yaeger KA, Rossitto CP, et al. "Staying home": early changes in patterns of neurotrauma in New York City during the COVID-19 pandemic. World Neurosurg. 2020;143:e344-e350.

2. Boserup B, McKenney M, Elkbuli A. The impact of the COVID-19 pandemic on emergency department visits and patient safety in the United States. Am J Emerg Med. 2020;38(9):1732-1736.

3. Hervey-Jumper SL, Li J, Lau D, et al. Awake craniotomy to maximize glioma resection: methods and technical nuances over a 27-year period. J Neurosurg. 2015;123(2):325-339.

4. Clamp PJ, Broomfield SJ. The challenge of performing mastoidectomy using the operating microscope with coronavirus disease 2019 personal protective equipment (PPE). J Laryngol Otol. 2020;134(8): 739-743.

5. Carobbio ALC, Filauro M, Parrinello G, et al. Microsurgical procedures during COVID-19 pandemic: the VITOM ${ }^{\otimes}$ 3D-HD exoscopic system as alternative to the operating microscope to properly use personal protective equipment (PPE). Eur Arch Otorhinolaryngol. 2020:;278(6):2129-2132.

6. Prachand VN, Milner R, Angelos P, et al. Medically necessary, timesensitive procedures: scoring system to ethically and efficiently manage resource scarcity and provider risk during the COVID-19 pandemic. J Am Coll Surg. 2020;231(2):281-288.

7. Bernhardt D, Wick W, Weiss SE, et al. Neuro-oncology management during the COVID-19 pandemic with a focus on WHO grade III and IV gliomas. Neuro Oncol. 2020;22:928-935.

8. Forrester JD, Nassar AK, Maggio PM, Hawn MT. Precautions for operating room team members during the COVID-19 pandemic. J Am Coll Surg. 2020;230(6):1098-1101.

9. Castelnuovo P, Turri-Zanoni M, Karligkiotis $A$, et al. Skull-base surgery during the COVID-19 pandemic: the Italian Skull Base Society recommendations. Int Forum Allergy Rhinol. 2020;10(8):963-967.

10. Flexman AM, Abcejo AS, Avitsian R, et al. Neuroanesthesia practice during the COVID-19 pandemic: recommendations from Society for Neuroscience in Anesthesiology and Critical Care (SNACC). J Neurosurg Anesthesiol. 2020;32(3):202-209.

11. Germanò A, Raffa G, Angileri FF, et al. Coronavirus disease 2019 (COVID-19) and neurosurgery: literature and neurosurgical societies recommendations update. World Neurosurg. 2020;139:e812-e817.

12. Jean WC, Ironside NT, Sack KD, et al. The impact of COVID-19 on neurosurgeons and the strategy for triaging non-emergent operations: a global neurosurgery study. Acta Neurochir (Wien). 2020;162(6):1229-1240.

13. Mohile NA, Blakeley JO, Gatson NTN, et al. Urgent considerations for the neuro-oncologic treatment of patients with gliomas during the COVID-19 pandemic. Neuro Oncol. 2020;22:912-917.

14. Mummaneni PV, Burke JF, Chan AK, et al. Consensus-based perioperative protocols during the COVID-19 pandemic. J Neurosurg Spine. 2020;34(1):13-21.

15. Pessina F, Navarria P, Bellu L, et al. Treatment of patients with glioma during the COVID-19 pandemic: what we learned and what we take home for the future. Neurosurg Focus. 2020;49(6):E10.

16. Zacharia BE, Eichberg DG, Ivan ME, et al. Letter: surgical management of brain tumor patients in the COVID-19 era. Neurosurgery. 2020;87(2):E197-E200.

17. Okunlola Al. Awake craniotomy in a COVID-19 positive patient: The challenges and outcome. Interdiscip Neurosurg. 2021;24:101064.

18. American Society of Anesthesiologists (ASA), Anesthesia Patient Safety Foundation (APSF), American Academy of Anesthesiologist Assistants (AAAA), American Association of Nurse Anesthetists (AANA). The Use of Personal Protective Equipment by Anesthesia Professionals during the COVID-19 Pandemic. Joint Position
Statement. Accessed April 4, 2021. https://www.asahq.org/-/media/ files/spotlight/

ppe-and-covid-joint-statement-asa-apsf-aaaa-anaa-june3-2020.pdf?la=en\&hash=AA7DCDF6104D28DA4C15057E3DB61 A884F4D00D0

19. Dolecek TA, Propp JM, Stroup NE, Kruchko C. CBTRUS statistical report: primary brain and central nervous system tumors diagnosed in the United States in 2005-2009. Neuro Oncol. 2012;14(suppl 5): v1-v49.

20. Ramakrishna R, Zadeh G, Sheehan JP, Aghi MK. Inpatient and outpatient case prioritization for patients with neuro-oncologic disease amid the COVID-19 pandemic: general guidance for neuro-oncology practitioners from the AANS/CNS Tumor Section and Society for Neuro-Oncology. J Neurooncol. 2020;147(3):525-529.

21. Khoury MN, Missios S, Edwin N, et al. Intracranial hemorrhage in setting of glioblastoma with venous thromboembolism. Neurooncol Pract. 2016;3(2):87-96.

22. Giovannini G, Pasini F, Orlandi N, et al. Tumor-associated status epilepticus in patients with glioma: clinical characteristics and outcomes. Epilepsy Behav. 2019;101(Pt B):106370.

23. Centers for Disease Control and Prevention. Framework for Healthcare Systems Providing Non- COVID-19 Clinical Care During the COVID-19 Pandemic. Accessed October 3, 2020. https://www.cdc. gov/coronavirus/2019-ncov/hcp/framework-non-COVID-care.html

24. Sanai N, Polley MY, McDermott MW, et al. An extent of resection threshold for newly diagnosed glioblastomas. J Neurosurg. 2011;115(1):3-8.

25. Stupp R, Mason WP, van den Bent MJ, et al. Radiotherapy plus concomitant and adjuvant temozolomide for glioblastoma. $N$ Engl J Med. 2005;352(10):987-996.

26. Noticewala SS, Ludmir EB, Bishop AJ, et al. Radiation for glioblastoma in the era of coronavirus disease 2019 (COVID-19): patient selection and hypofractionation to maximize benefit and minimize risk. Adv Radiat Oncol. 2020;5(4):743-745.

27. Sun R, Achkar S, Ammari S, et al. Systematic screening of COVID19 disease based on chest CT and RT-PCR for cancer patients undergoing radiation therapy in a coronavirus French hotspot. Int $\mathrm{J}$ Radiat Oncol. Published online February 17, 2021. doi:10.1016/ j.jrobp.2021.02.022

28. Yanagihara TK, Holland RE, Chera B. Practical challenges of mask-to-mask encounters with patients with head and neck cancers amid the coronavirus disease 2019 pandemic. Adv Radiat Oncol. 2020;5(4):651-655.

29. Roy A, Andruska N, Orlowski HLP, et al. The novel use of a commercially available video-conference platform to facilitate multidisciplinary target volume review and delineation for skull-base radiation therapy during the coronavirus disease 2019 pandemic. Adv Radiat Oncol. 2021;6(2):100598.

30. Romani C, Conlon M, Oliver M, et al. The operation of Canada's only virtually operated radiation oncology service during the COVID-19 pandemic. Adv Radiat Oncol. 2020;6(3):100634.

31. Chen $W, S u X Y$, Wang VJ, et al. Novel coronavirus international public health emergency: guidance on radiation oncology facility operation. Adv Radiat Oncol. 2020;5(4):560-566.

32. Lamborn KR, Chang SM, Prados MD. Prognostic factors for survival of patients with glioblastoma: recursive partitioning analysis. Neuro Oncol. 2004;6(3):227-235.

33. Núñez-Velasco S, Mercado-Pimentel R, Rodríguez-Arias R. Letter to the editor: awake craniotomy for intracranial gliomas during coronavirus disease 2019 pandemic. World Neurosurg. 2020;140: 470-472.

34. Celtikci E, Karaaslan B, Börcek $A O ̈$, Emmez OH. Reduced field of view under the surgical microscope due to personal protective equipment: lessons learned during the COVID-19 pandemic. Neurosurg Focus. 2020;49(6):E15. 
35. Lokesh PK, Chowdhary S, Pol SA, et al. Quantification of biomaterial dispersion during otologic procedures and role of barrier drapes in COVID 2019 era: a laboratory model. J Laryngol Otol. 2020;134(11):1-6.

36. Lawrence RJ, O'Donoghue GM, Kitterick P, Hartley DEH. Use of a novel drape 'tent' as an infection prevention control measure for mastoid surgery. J Laryngol Otol. 2020;134(12):1115-1117.

37. Zoabi T, Ronen $\mathrm{O}$. A novel technique for protecting staff during microlaryngoscopy procedures. J Laryngol Otol. 2021;135(1): 83-85.

38. Das A, Mitra S, Kumar S, Sengupta A. Two-drape closed pocket technique: minimizing aerosolization in mastoid exploration during COVID-19 pandemic. Eur Arch Otorhinolaryngol. 2020;277(12): 3529-3532.

39. Ghazali N, Attardo G, Markose G. Modification of the microscope drape to provide a closed surgical field in transoral robotic surgery. Br J Oral Maxillofac Surg. 2020;58(7):867-868.

40. David AP, Jiam NT, Reither JM, et al. Endoscopic skull base and transoral surgery during COVID-19 pandemic: minimizing droplet spread with negative-pressure otolaryngology viral isolation drape. Head Neck. 2020;42(7):1577-1582.

41. Francom CR, Javia LR, Wolter NE, et al. Pediatric laryngoscopy and bronchoscopy during the COVID-19 pandemic: a four-center collaborative protocol to improve safety with perioperative management strategies and creation of a surgical tent withdisposable drapes. Int J Pediatr Otorhinolaryngol. 2020;134:110059.

42. D’Amico RS, Khatri D, Kwan K, et al. Coronavirus neurosurgical/ head and neck drape to prevent aerosolization of coronavirus disease 2019 (COVID-19): the Lenox Hill Hospital/Northwell Health solution. World Neurosurg. 2020;142:314-317.
43. loannidis D, Tsagkovits A, Rokade A. Minimising aerosol spread during endoscopic sinus and skull base surgery. Experimental model evaluation of the efficacy of the microscope drape method. J Laryngol Otol. 2020;134(9):1-7.

44. Gordon SA, Deep NL, Jethanamest D. Exoscope and personal protective equipment use for otologic surgery in the era of COVID-19. Otolaryngol Head Neck Surg. 2020;163(1):179-181.

\section{Disclosures}

Dr. Kotecha reported personal fees from Elekta AB, Accuray, Inc., Novocure, Inc., Elsevier, Inc., and Viewray, Inc. outside the submitted work.

\section{Author Contributions}

Conception and design: Alvarez, McDermott. Acquisition of data: Alvarez, Siomin. Analysis and interpretation of data: McDermott, Siomin. Drafting the article: Alvarez, Kotecha, McDermott. Critically revising the article: all authors. Reviewed submitted version of manuscript: all authors. Approved the final version of the manuscript on behalf of all authors: Alvarez. Study supervision: Siomin.

\section{Correspondence}

Reinier Alvarez: University of Colorado School of Medicine, Aurora, CO. reinier.alvarez@cuanschutz.edu. 